Gut, 1979, 20, 992-996

\title{
Peroperative transduodenal biopsy of the pancreas
}

\author{
D. E. F. TWEEDLE \\ From the Department of Surgery, University Hospital of South Manchester, Manchester
}

SUMMARY Peroperative transduodenal biopsy of the pancreas was performed in 65 patients with the disposable Trucut needle. The technique described is simple, effective, and may reveal unsuspected malignancy. A histological diagnosis of malignancy is reliable but false negative diagnoses may arise because of failure to obtain representative samples.

Although the use of such techniques as endoscopic retrograde pancreatography and computer assisted tomography provides valuable preoperative information in patients with pancreatic disease (Cotton et al., 1978), most surgeons would prefer to have histological confirmation of the provisional diagnosis before performing a major resection. However, many of them shun this procedure because of the mortality and morbidity associated with it. The complications include haemorrhage, acute pancreatitis, fistulae, pseudocysts, and subphrenic abscesses. Since 1957 the published mortality associated with pancreatic biopsy has varied from 0 to $35 \%$ (Williams et al., 1960; George et al., 1975). However, the true mortality due to biopsy alone has been difficult to ascertain, as in many of the reports additional operative procedures involving the paricreas were performed at the same time.

The accuracy of diagnosis, complications, and mortality associated with transduodenal biopsy of the head of the pancreas using a commercial, disposable needle of the cutting type (Trucut needle, Baxter Travenol Ltd) have been assessed between January 1972 and June 1978 in 65 patients in whom further operative procedures involving the pancreas were not required or were considered inappropriate because of the patient's age or condition.

\section{Methods}

The laparotomy was performed through a Kocher's subcostal incision. The duodenum was mobilised by Kocher's manoeuvre and any palpable lesion in the head of the pancreas was located with the tip of the finger of the left hand. The needle was inserted into the duodenum and then directed at the target palpated with the left hand. The left hand was then

Received for publication 25 May 1979 removed to steady the needle and the outer cutting cannula was advanced to cut the core of tissue. Occasionally an adequate biopsy was not obtained at the first attempt and the procedure was repeated until a satisfactory biopsy was obtained. The puncture site in the duodenum was oversewn and a suction drain was left in the hepatorenal pouch. Initially biopsies were performed in patients with suspected carcinoma of the pancreas or chronic pancreatitis. However, as confidence in the safety of the procedure increased, biopsies were taken from patients who had suffered attacks of pancreatitis associated with biliary disease.

\section{Results}

The results of examination of the paraffin sections were classified into three types of tissue (Figure): (a) normal pancreas, (b) pancreatic fibrosis or inflammation, (c) pancreatic carcinoma.

In the main, the pathologists who reported these biopsies were satisfied with the material obtained. The low power view shown in the Figure demonstrates the core of tissue obtained by this technique. Pancreatic tissue was obtained in 62 of the 65 patients. Of the three patients in whom pancreatic tissue was not obtained, in two the biopsy contained duodenal mucosa only and in the third patient the biopsy contained renal tissue. The results of the 62 biopsies have been subdivided into four categories and are shown in Table 1. Histological confirmation of suspected malignancy was obtained in 23 out of 28 patients. Of the remaining five patients one is alive and well with no evidence of malignant disease two years after his laparotomy for obstructive jaundice, which presumably was due to inflammation in the pancreas. However, the remaining four patients have subsequently died from malignant disease and the findings at laparotomy leave no reason for doubt as to the site of origin of the tumour. Of six patients with 
Table 1 Comparison of histological diagnoses from biopsy specimens and ultimate clinical diagnosis

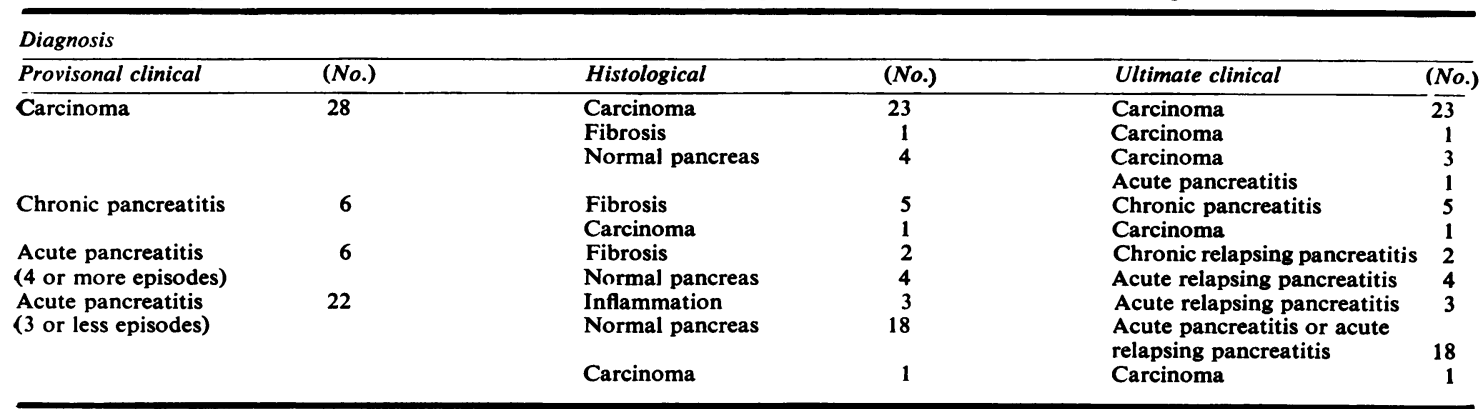

suspected chronic pancreatitis, fibrosis was confirmed in five and an unsuspected malignancy diagnosed in one patient. Six patients had suffered four or more episodes of acute pancreatitis and the biopsy specimen contained normal pancreatic tissue in four. In two of these patients the tissue showed the fibrotic changes typical of chronic pancreatitis and, according to the Marseilles Convention, these patients should be classified as suffering from chronic relapsing pancreatitis. Twenty-two patients had suffered from acute pancreatitis on three or less occasions. Three of the specimens showed inflammatory changes which had not progressed to fibrosis but the majority (which were performed during a quiescent phase) showed no abnormality. In one patient an unsuspected malignancy in the head of the pancreas was diagnosed, the gland being acutely inflamed distal to this.

\section{Complications}

None of the patients died as a direct result of the biopsy, but one patient with malignant disease died from renal failure in the postoperative period. The complications of acute pancreatitis, pseudocyst, fistula, and subphrenic abscess reported by others were not observed. Minor haemorrhage occurred in five patients: in two, brisk arterial haemorrhage into the duodenal lumen was controlled by simple compression of the head of the pancreas, and small subserosal haematomata of the duodenal wall were controlled by the same conservative measures in three patients. In none of the patients was there evidence of extraluminal haemorrhage in the postoperative period.

The only complication of note in this series was the development of haematuria in the patient whose biopsy specimen contained renal tissue. This settled spontaneously after 24 hours and, although there were areas in the biopsy specimen which contained inflammatory changes, an intravenous urogram showed no abnormality and the urine has remained sterile on culture.

\section{Discussion}

In 65 attempts at transduodenal biopsy, adequate biopsies were obtained on 62 occasions $(93 \%)$. Initially a $15.2 \mathrm{~cm}(6$ in) needle was used but more recently the $7.6 \mathrm{~cm}$ ( $3 \mathrm{in}$ ) needle has been used with greater ease. Unfortunately, both hands must be applied to the handle of the needle during the cutting motion. The position of the tip of the needle may alter when the left hand is withdrawn from behind the head of the pancreas and care must be taken to ensure that the tip of the needle is not advanced when the biopsy is taken. It is thought that both these mechanisms may have been responsible for the inadvertent renal biopsy. Of the complications reported by others, fistula and abscess formation have been particularly troublesome, presumably because of leakage of pancreatic enzymes from the biopsy site (Spjut and Ramos, 1957; Schultz and Sanders, 1963; Lund, 1969; British Medical Journal, 1975). Transduodenal biopsy of the head of the pancreas using the diathermy or an ophthalmic trephine has been suggested as a convenient method of obtaining a biopsy which will ensure that any leakage occurs into the lumen of the bowel (Smith, 1953). This commercially available and disposable needle (size 14G) proved to be admirable in this respect, there being no evidence of extraluminal leakage in any of the patients. Although aspiration biopsy may be performed by the same method using a smaller needle (size 18G), the tissue obtained is amorphous (Kline and Neil, 1975) and does not allow the pathologist to study the architecture of the gland as is possible by this technique as shown in the Figure.

Although endoscopic retrograde cholangiopancreatography, percutaneous transhepatic cholangiography, and computer assisted tomography have proved to be a great boon to the surgeon operating on patients with obstructive jaundice, the facilities and expertise required for these investigations are, unfortunately, not available in the majority of hospitals in Great Britain and the results of these 

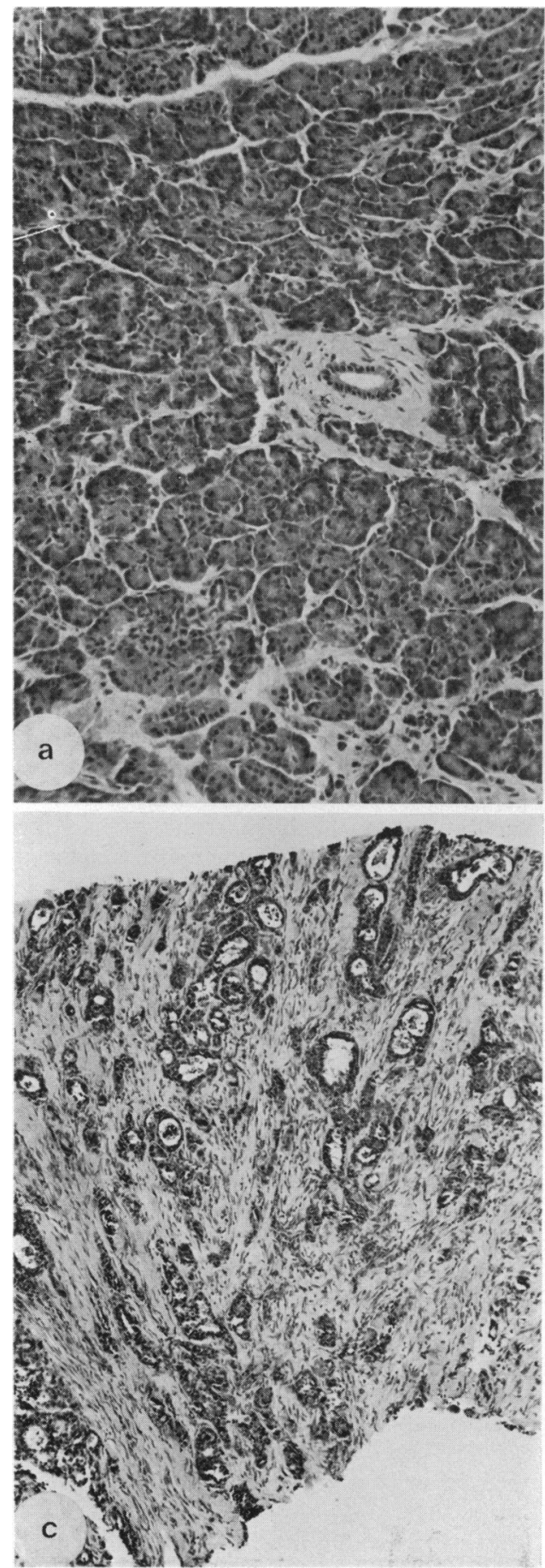

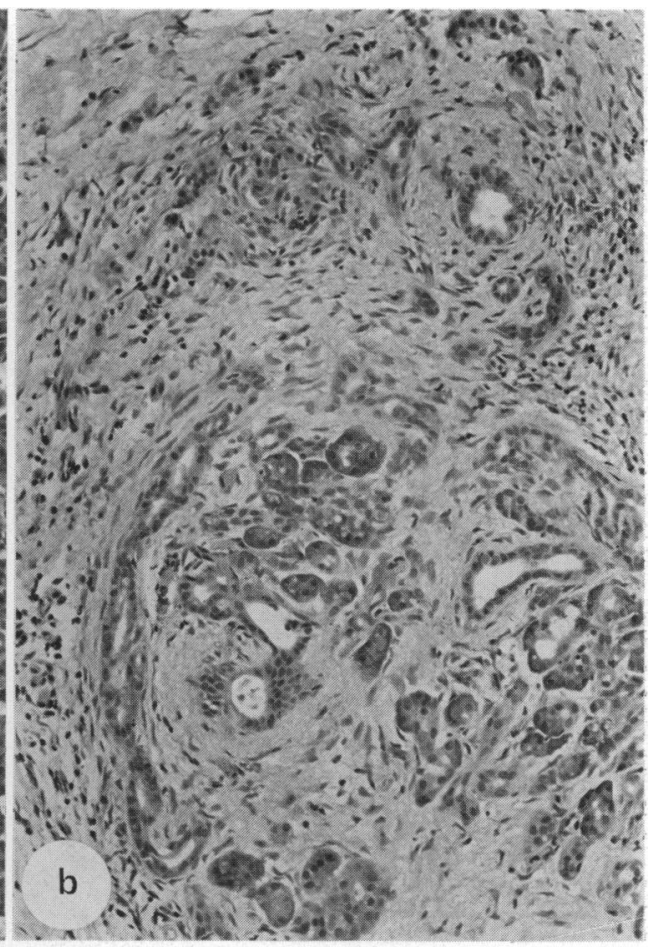

Figure Typical histological specimens obtained by needle biopsy (stained with haematoxylin and eosin). (a) Normal pancreas (original magnification $x 205$ ). (b) Pancreatic fibrosis (original magnification $x$ 205). (c) Pancreatic carcinoma (original magnification $x 80$ ). 
investigations are occasionally misleading. In the future percutaneous needle biopsy of the pancreas under ultrasonic guidance may prove to be the most useful preoperative investigation in patients with pancreatic-disease (Hancke et al., 1975), but it is likely to be many years before this technique will be available in most hospitals. Even in institutions in which these sophisticated investigations are readily available, the surgeon may still be required to perform an urgent laparotomy for obstructive jaundice without adequate preoperative investigation.

The major use of pancreatic biopsy is in the differential diagnosis of chronic pancreatitis and carcinoma. In the 34 patients with suspected malignancy or chronic pancreatitis there were no false positive diagnoses in 24 patients with histological evidence of malignancy. However, there were four false negative diagnoses in 10 patients with no evidence of malignancy in the biopsy. In this series of patients malignancy was correctly diagnosed in 25 out of 29 patients $(86 \%)$. The results of biopsy in carcinoma of the pancreas from various centres are shown in Table 2. It would appear that diagnostic accuracy has increased during the last 20 years, and the most recent results show some uniformity, but it is evident that false negative diagnoses may be made and the surgeon must be aware of this possibility.

Table 2 Carcinoma of pancreas-results of biopsy

\begin{tabular}{|c|c|c|c|}
\hline & Number & $\begin{array}{l}\text { Positive } \\
\text { diagnosis }\end{array}$ & $\%$ \\
\hline Spjut and Ramos (1957) & 68 & 53 & 78 \\
\hline Coté et al. (1959) & 109 & 62 & 57 \\
\hline Williams et al. (1960) & 43 & 37 & 86 \\
\hline Schultz and Sanders (1963) & 159 & 103 & 65 \\
\hline Forsgren et al. (1968) & 50 & 48 & 96 \\
\hline Lund (1969) & 55 & 44 & 80 \\
\hline Arnesjo et al. (1972) & 18 & 17 & 95 \\
\hline Koivuniemi et al. (1972) & 18 & 16 & 89 \\
\hline Kline and Neal (1975) & 18 & 17 & 95 \\
\hline George et al. (1975) & 35 & 31 & 88 \\
\hline Tweedle (1979) & 29 & 25 & 86 \\
\hline
\end{tabular}

Many surgeons feel that excisional surgery for carcinoma of the pancreas cannot be justified by the poor results. Nevertheless, the ability to establish the correct diagnosis is important. The prognosis is very different for patients suffering from carcinoma of the pancreas, lower common bile duct, ampulla of Vater, and of the duodenum (Warren et al., 1975).

At a symposium in Marseilles (Sarles, 1963) pancreatitis was classified into four categories: (1) acute pancreatitis, (2) acute relapsing pancreatitis, (3) chronic relapsing pancreatitis, and (4) chronic pancreatitis. Patients suffering from acute relapsing pancreatitis or chronic relapsing pancreatitis are indistinguishable during acute attacks, the two groups being differentiated by a histological and clinical return to normality in patients with acute relapsing pancreatitis. It has been suggested that it is very uncommon for acute pancreatitis to progress to chronic pancreatitis (Pollock, 1959; Sarles and Camatte, 1963; Trapnell, 1966) and in patients suffering from acute relapsing pancreatitis and biliary tract disease pancreatitis is usually cured by surgical correction. Although uncommon, patients have been seen who continued to suffer from acute relapsing pancreatitis after biliary surgery, in whom there was evidence of inflammation and fibrosis in the pancreas. Consequently, when confidence in the safety in the technique had been established, pancreatic biopsies were taken from all patients undergoing biliary surgery who had suffered from acute pancreatitis. Five of the 28 patients had inflammation with or without fibrosis in the biopsy and it appears that repeated attacks of pancreatitis in patients with biliary tract disease can produce irreversible pancreatic damage. The incidence and the severity of these changes was greater in those patients who had suffered four or more episodes of acute pancreatitis than in those who had suffered three or less attacks, suggesting that the changes are progressive and related to the number of attacks. However, although these patients had histological evidence of pancreatic disease, none of them had signs or symptoms of pancreatic disease between the acute exacerbations. Pancreatic disease is a common cause of symptoms after cholecystectomy (Raymer et al., 1960) and it is hoped that the information obtained by peroperative biopsy will prove of value in the subsequent management of these patients.

I thank the many pathologists who examined the biopsies and in particular $\operatorname{Dr} M$. Harris, senior lecturer in pathology in the University of Manchester, who prepared the photomicrographs.

\section{References}

Arnesjö, B., Stormby, N., and Ảkerman, M. (1972). Cytodiagnosis of pancreatic lesions by means of fineneedle biopsy during operation. Acta Chirurgica Scandinavica, 138, 363-369.

British Medical Journal (1975). Pancreatic biopsy. (Leading article). British Medical Journal, 2, 354.

Coté, J., Dockerty, M. B., and Priestley, J. T. (1959). An evaluation of pancreatic biopsy with the Vim-Silverman needle. Archives of Surgery, 79, 588-596.

Cotton, P. B., Denyer, M. E., Kreel, L., Husband, J., Meire, H. B., and Lees, W. (1978). Comparative clinical impact of endoscopic pancreatography, greyscale ultrasonography, and computed tomography (EMI scanning) in pancreatic disease: preliminary report. Gut, 19, 679. 
Forsgren, L., Hansson, K., Lundh, G., and Nordenstam, H. (1968). Pancreatic biopsy. Acta Chirurgica Scandinavica, 134, 457-460.

George, P., Brown, C., and Gilchrist, J. (1975). Operative biopsy of the pancreas. British Journal of Surgery, 62 , 280-283.

Hancke, S., Holm, H. H., and Koch, F. (1975). Ultrasonically guided percutaneous fine needle biopsy of the pancreas. Surgery, Gynecology and Obstetrics, 140, 361-364.

Kline, T. S., and Neal, H. S. (1975). Needle aspiration biopsy. American Journal of Clinical Pathology, 63, 16-19.

Koivuniemi, A., Lempinen, M., and Pantzar, P. (1972). Fine needle aspiration biopsy of pancreas. Annales Chirurgiae et Gynaecologiae Fenniae, 61, 273-280.

Lund, F. (1969). Carcinoma of the pancreas. Acta Chirurgica Scandinavica, 135, 515-517.

Pollock, A. V. (1959). Acute pancreatitis. British Medical Journal, 1, 6-14.

Raymer, J. B., Tarpinian, D. A., and Meyers, S. G.
(1960). Symptoms following cholecystectomy. American Journal of Digestive Diseases, 5, 55-62.

Sarles, H., and Camatte, R. (1963). Pancreatites Aiguës: Conceptions et Thérapeutiques Récentes. Masson: Paris.

Schultz, N. J., and Sanders, R. J. (1963). Evaluation of pancreatic biopsy. Annals of Surgery, 158, 1053-1057.

Smith, R. (1953). In The Surgery of Pancreatic Neoplasms, p. 51. Livingstone: Edinburgh.

Spjut, H. J., and Ramos, A. J. (1957). An evaluation of biopsy-frozen section of the ampullary region and pancreas: a report of 68 consecutive patients. Annals of Surgery, 145, 923-930.

Trapnell, J. E. (1966). The natural history and prognosis of acute pancreatitis. Annals of the Royal College of Surgeons, 38, 265-287.

Warren, K. W., Choe, D. S., Plaza, J., and Relihan, M. (1975). Results of radical resection for periampullary cancer. Annals of Surgery, 181, 534-540.

Williams, R. D., Elliott, D. W., and Zollinger, R. M. (1960). Surgery for malignant jaundice. Archives of Surgery, 80, 992-997. 